\title{
Improvement in direction discrimination: No role for eye movements
}

\author{
WILLIAM KOSNIK, JOHN FIKRE, and ROBERT SEKULER \\ Northwestern University, Evanston, Illinois
}

\begin{abstract}
Practice improves observers' ability to discriminate between highly similar directions of motion. In an effort to clarify the basis for this improvement, we recorded an observer's eye movements while he made direction discriminations. We found that the observer did not need to track the moving target in order to learn the discrimination. Both at the beginning and at the end of training the observer's eye movements more closely resembled movements made while fixating a stationary target, and did not at all resemble movements made while intentionally tracking the stimulus. These results suggest that the learned discrimination of the direction of moving targets is perceptual in nature and does not depend on the learning of a sensorimotor response.
\end{abstract}

Practice improves an observer's ability to discriminate one direction of movement from another, highly similar, direction of movement (Ball \& Sekuler, 1982). This improvement in discrimination has two noteworthy features, directional selectivity and persistence. More particularly, the improvement is restricted to directions that are similar to the one with which the observer has practiced, and the improvement endures for several months without noticeable decrement. In the present experiment, we sought to clarify the origin of this direction-specific change in discrimination.

Basically, there are two possible routes by which improved direction discrimination might be achieved. The route may be purely visual, possibly reflecting changes in the selectivity of neurons at some stage of the visual system. Alternatively, the route may be a sensorimotor one, with the observer learning to use tracking eye movements to discriminate between two directions.

In their original study, Ball and Sekuler (1982) did measure the eye movements of 2 observers and found steady fixation with high levels of performance. However, significant questions about the role of eye movements remained unanswered. Because their recording system could not resolve eye movements smaller than approximately $45^{\prime}$ of arc, Ball and Sekuler were unable to rule out the possibility of small, but visually significant, eye movements. This possibility is important because the stimulus duration they used, $500 \mathrm{msec}$, might prevent very large pursuit movements anyway. More importantly, though, they neglected to record eye movements at different stages of training. Therefore, it remains possible that changes in eye movements may have played some role in the observed change in performance.

We decided to investigate sensorimotor contributions to direction discrimination more thoroughly by analyz-

This work was supported by Grant AFOSR 80-0246 from the Air Force Office of Scientific Research. The authors' address is Cresap Neuroscience Laboratory, Northwestern University, Evanston, IL 60201. ing an observer's eye movements at the beginning and end of training, using an eye tracking device that is capable of resolving movements of about $1^{\prime}$ of arc.

\section{METHOD}

\section{Observer}

The observer was a 20 -year-old male who had never participated in a psychophysical study before. He was paid for his participation, and to ensure high motivation, he received an additional $\$ .01$ for every correct response. The observer viewed the stimulus display with the right eye; the other eye was occluded with an opaque patch.

\section{Apparatus}

The experimental set-up was similar to that used by Ball and Sekuler (1982). The stimuli were spatially random dots moving in a uniform direction at $10 \% \mathrm{sec}$ across the face of a cathode ray tube (CRT). On average, 322 dots were visible within a circular aperture of $5^{\circ}$ diameter. The dots, which had a luminance of $104 \mathrm{~cd} / \mathrm{m}^{2}$, were easily visible against the CRT's luminance of $2.06 \mathrm{~cd} / \mathrm{m}^{2}$. A small fixation point was provided in the center of the screen.

\section{Procedure}

A trial consisted of two stimulus presentations, each lasting $640 \mathrm{msec}$ (except on the first day of training, when each presentation lasted $512 \mathrm{msec}$ ). ${ }^{1}$ The two presentations were separated by an interval of $1.25 \mathrm{sec}$, during which the CRT was blank.

The directions of movement of the dots within the two presentations were either the same [in both presentations the dots moved in an upward direction $\left(90^{\circ}\right.$ from horizontal)] or different [during one interval the dots moved upward; in the other interval the dots moved either $3^{\circ}$ to the left $\left(93^{\circ}\right)$ or $3^{\circ}$ to the right $\left(87^{\circ}\right)$ of $90^{\circ}$ upward]. Same and Different trials were randomly presented with equal probability. For Different trials the computer randomized whether the upward movement would occur in the first interval or in the second; the two nonupward directions, $87^{\circ}$ and $93^{\circ}$, occurred randomly, but equally often.

After each trial the observer judged whether the two directions had been the same or different, that is, whether both stimuli moved in the upward direction or whether one moved in the upward direction and the other moved in a direction other than upward. A computer-generated tone told the observer whether or not his response was correct.

Training comprised an extended series of discrimination trials in blocks of 32 trials each. Because half of the trials were Same 
and half Different, and because there were two stimulus presentations per trial, every block of 32 trials yielded 64 stimulus presentation intervals -48 in which movement was upward, 8 in which movement was in the direction of $87^{\circ}$ degrees, and 8 in which movement was in the direction of $93^{\circ}$ degrees.

On the first day of training, 4 blocks of 32 trials were run. On subsequent days, 10 blocks of 32 trials each were run. A rest was given after each block. Training was spread out over 8 days.

\section{Eye Movement Recording}

Two-dimensional eye movements were measured from the observer's right eye by a Scientific Research International (SRI) dual Purkinje Image Eye Tracker (Mark IV; Crane \& Steele, 1978). A calibration procedure determined the Eye Tracker's gain and noise levels. Noise levels were $0.43^{\prime}$ of arc in the horizontal channel and $0.40^{\prime}$ of arc in the vertical channel. Thus, movement of $1^{\prime}$ of arc could be reliably measured.

Eye position records obtained from four blocks of trials on the first day of discrimination training were digitized at a rate of $500 \mathrm{~Hz}$ and stored in computer memory. A $500-\mathrm{Hz}$ sampling rate was used in order to accommodate the full $200-\mathrm{Hz}$ bandwidth of the recording instrument. Eye positions were collected throughout the 512msec stimulus presentation. This yielded one eye position record of 256 data points per stimulus presentation.

On subsequent training days, a $640-\mathrm{msec}$ stimulus presentation interval was used. This change was necessitated by the introduction of a low-pass filter in the data collection system on the second training day, as explained below.

On the last day of training, we measured eye positions during the last five blocks of discrimination training. The data were lowpass filtered at $50 \mathrm{~Hz}(-36 \mathrm{~dB} /$ octave $)$ prior to being digitized at a rate of $100 \mathrm{~Hz}$. These recording parameters required a $640-\mathrm{msec}$ stimulus presentation, but resulted in a considerable savings in computer storage without loss of significant eye position information. Thus, each eye position record collected on the last day of training contained 64 data samples.

After the data were collected and stored, each eye position record was edited for eyeblinks and other artifacts. If an eyeblink occurred, or if tracking had been interrupted at any time during a record, the entire record was omitted from the analysis. In addition, a record was eliminated if it contained a saccade whose velocity was greater than $30 \%$ sec.

\section{RESULTS}

\section{Discriminability}

The observer's discrimination performance on each block of trials was expressed in units of d' (Swets, 1964), computed from the proportion of Different trials correctly identified as different (that is, hits) and the proportion of Same trials incorrectly identified as different (that is, false alarms). A discriminability score for one day was obtained by averaging across all blocks of trials run on that day.

The first question to be answered was whether the observer's discrimination performance changed with practice, and, if it did, whether such changes mirrored those previously reported by Ball and Sekuler (1982). We have displayed in Figure 1 the observer's discrimination performance over the 8 days of training. To facilitate comparison, we have also plotted Ball and Sekuler's results, which represent the average performance of 8 observers. Note the similarity of the two curves, each demonstrating a steady improvement in performance and reaching the same high level of discrimination.

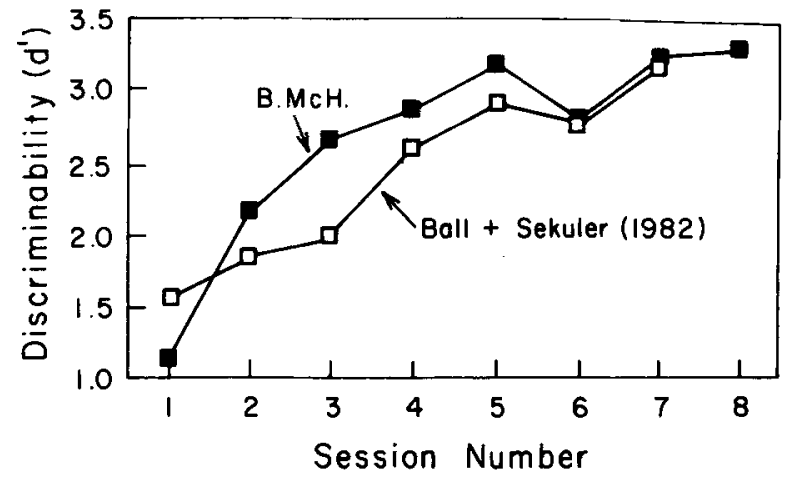

Figure 1. Discriminability (d') of the direction of a moving target as a function of the number of training days. Performance of the observer in this study (B. McH.) is compared with the average performance of 8 observers in Ball and Sekuler's (1982) study.

\section{Eye Movements: Orientation}

Next we wanted to determine whether the improvement in discrimination was mediated by the observer's having learned to track the stimulus. Because tracking eye movements would cause successive samples of eye position to lie along a straight line, we developed an estimate of the main axis along which the eyes moved during each stimulus presentation. We called this estimate the dominant orientation. To obtain this dominant orientation, the eye positions recorded during a presentation interval were represented in two dimensions and a least squares regression line was fit thereto. The slope of this line, expressed in degrees from the $0^{\circ}$ meridian, defined the dominant orientation of the eye movements.

To illustrate this procedure, two eye position records are shown in Figure 2; the dominant orientations have been drawn through the sampled eye positions. For each record, the $F$ ratio associated with the regression coefficient is highly significant: $F(1,253)=898.6$ for the top record, and $F(1,253)=110.4$ for the bottom record, both ps $<.0001 .^{2}$

Table 1 gives the mean dominant orientation of the eye positions recorded during the first and last days of training. These dominant orientations have been sorted according to stimulus direction, with the $87^{\circ}$ direction in the first column, the $90^{\circ}$ direction in the second, and the $93^{\circ}$ direction in the third. Measurements made at the beginning of training are represented in the top row and measurements from the end of training are represented in the middle row. Note that orientations are expressed as axial values, meaning that $0^{\circ}$ and $180^{\circ}$ are equivalent to one another. Orientations take on values from $0^{\circ}$ to $179^{\circ}$. Means and variances were computed using statistics for directional data (Mardia, 1972).

As seen in the top row of Table 1, the mean dominant orientation for records at the beginning of training was centered near the horizontal axis $\left(0^{\circ}-180^{\circ}\right)$ for all three stimulus directions. Also note that there was no correspondence between the change in direction of the stimulus 

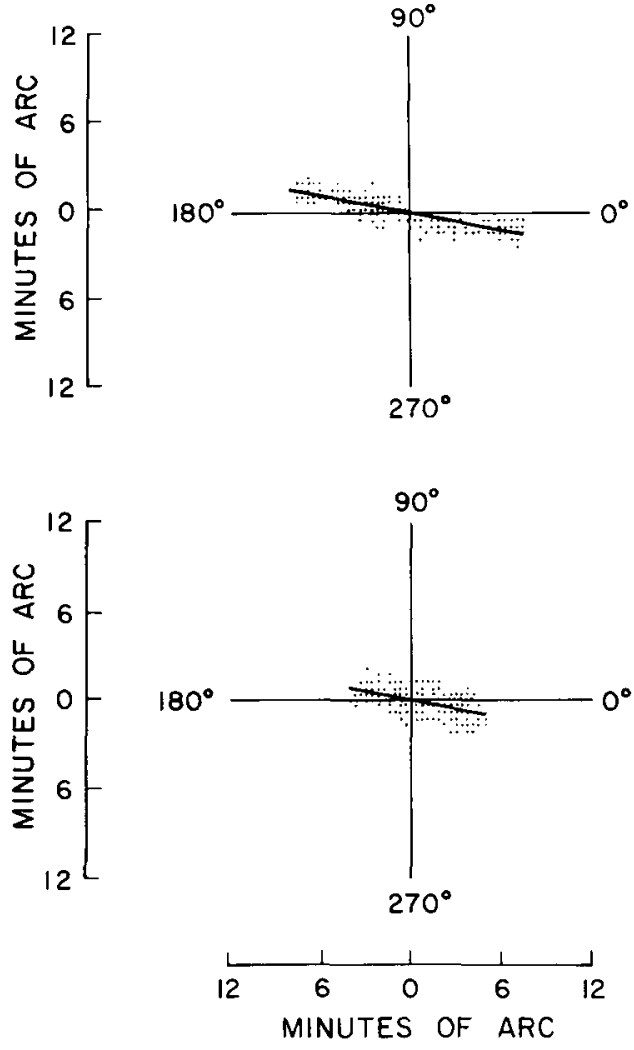

Figure 2. A two-dimensional eye position record collected during one stimulus presentation is displayed in each panel. A least squares regression line is fit to each record and represents the dominant axial orientation of the eye position record. The length of the regression line defines the hypothetical distance the eye moved along its dominant orientation; the calculation of this distance is described in the text. Note that, although the two records are well described by a straight line, the record in the top panel departs significantly from a linear model, whereas the record in the bottom panel is completely described by a linear model (see Note 1).

movement and the dominant orientation. A change in stimulus direction from $90^{\circ}$ to $87^{\circ}-\mathrm{a}$ shift of $3^{\circ}$ to the right-was not accompanied by a corresponding change in the dominant orientation. Instead, the dominant orientation shifted from $168^{\circ}$ to $176^{\circ}$, a net change of $8^{\circ}$ to the left. A change in stimulus direction from $90^{\circ}$ to $93^{\circ}-$ a shift of $3^{\circ}$ leftward-also failed to elicit a corresponding change in dominant orientation. In this case, the dominant orientation shifted $6^{\circ}$ to the right.

An examination of the middle row of Table 1 shows no better correspondence between stimulus direction and dominant orientation at the end of training. Again, the mean dominant orientation at the end of training was close to the horizontal axis for all three stimulus directions. In response to stimulus movement of $90^{\circ}$, the dominant orientation was $24^{\circ}$. A change in stimulus direction of $3^{\circ}$ to the left or right of vertical was not followed by a similar change in dominant orientation. In fact, the mean dominant orientation was $17^{\circ}$ for both off-vertical stimulus directions.
So that the reader can better appreciate the variability in the obtained dominant orientations, we show in Figure 3 the distribution of the dominant orientations cumulated over presentation intervals. These are the distributions that Table 1 summarizes. The upper portion of Figure 3 portrays data collected at the beginning of training; the middle portion portrays data from the end of training. Each column represents one direction of stimulus movement: $87^{\circ}, 90^{\circ}$, or $93^{\circ}$.

Note that for neither the beginning nor the end of training is there any obvious systematic relation between the dominant orientations and the direction of the stimulus movement. Moveover, there is no systematic change in the distribution of dominant orientations from beginning to end of training.

\section{Eye Movements: Magnitude}

Having characterized the dominant orientations of the eye position records, we wanted to determine the linear distances the eye traveled along the dominant orientations. The magnitude of the dominant orientation was measured as the length of the regression line. The endpoints of the regression line were determined by first measuring one of the values-either the $x$ or the $y$ value-from each coordinate pair that defined the maximum and minimum points of the eye positions along one axis. The other value of each coordinate pair was estimated from the regression equation. Thus, each pair of coordinates that determined the endpoints of the regression line consisted of a real value and an estimated value. The distance between the two pairs of coordinates defined the magnitude of the dominant orientation of the eye position record. The lengths of the lines of best fit in Figure 2 have been drawn to correspond with this definition.

Table 2 lists the mean magnitudes of the dominant orientations for all eye position records from a given day of training. The format is the same as that of Table 1. Both at the beginning and at the end of training, just $1^{\prime}$ of arc separated the dominant magnitudes associated with the three stimulus directions. The direction of movement of the stimulus did not influence the magnitude of the eye movements. Furthermore, only $l^{\prime}$ ' of arc distinguished the

Table 1

Mean Dominant Axial Orientations of the Eye Position Records (in Degrees)

\begin{tabular}{lccc} 
& \multicolumn{3}{c}{ Stimulus Direction } \\
\cline { 2 - 4 } Session & $87^{\circ}$ & $90^{\circ}$ & $93^{\circ}$ \\
\hline Beginning of & 176 & 168 & 162 \\
Training & $(18.5)$ & $(20.3)$ & $(19.8)$ \\
& {$[28]$} & {$[172]$} & {$[27]$} \\
End of & 17 & 24 & 17 \\
Training & $(26.5)$ & $(27.3)$ & $(19.8)$ \\
& {$[40]$} & {$[235]$} & {$[40]$} \\
Intentional & 79 & 88 & 91 \\
Tracking & $(2.67)$ & $(2.39)$ & $(1.30)$ \\
& {$[8]$} & {$[35]$} & {$[5]$} \\
\hline
\end{tabular}

Note-Standard deviations are shown in parentheses; the number of orientations included in each mean is shown in brackets. 
STIMULUS DIRECTION

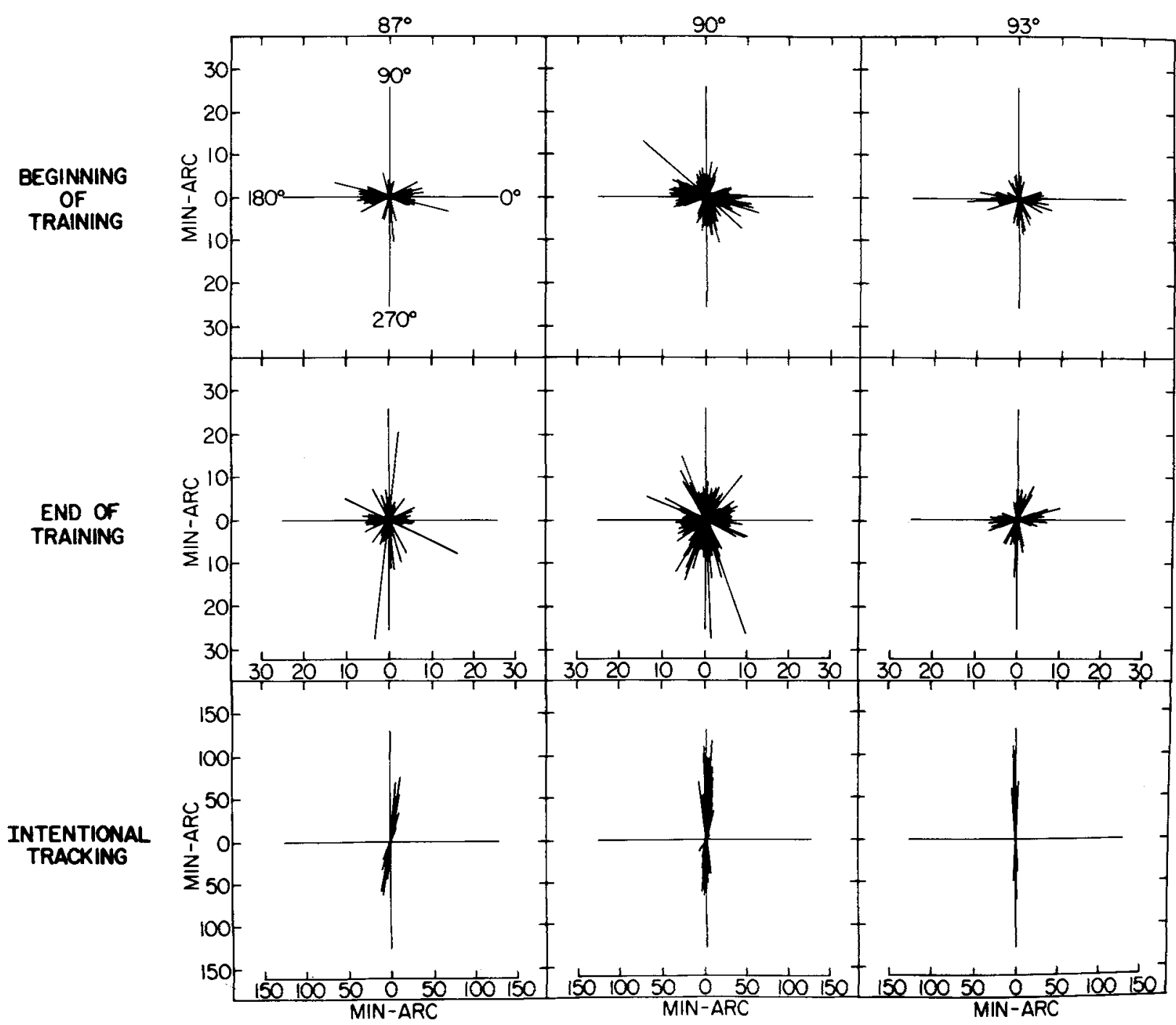

Figure 3. Distributions of the dominant axial orientations of the eye position records, arranged according to recording session and direction of stimulus movement. Magnitudes of the orientations are also shown. Note that magnitudes for the beginning and end of training are plotted on a scale of $60^{\prime}$ of arc; magnitudes recorded during intentional tracking of the stimulus are plotted on a scale of 300' of arc.

mean dominant magnitude at the beginning of training from the comparable value at the end of training.

Note that across all stimulus directions and across days of training, the mean dominant magnitude was very much smaller than the distance traveled by the stimulus on either the first or last day of training (5.1 $1^{\circ}$ on the first day and $6.4^{\circ}$ on the last day). The distributions of magnitudes associated with each stimulus direction are shown in the top and middle portions of Figure 3. These magnitudes range from $5.4^{\prime}$ to $50.0^{\prime}$ of arc.

To further characterize the eye movements made during discrimination training, we measured eye movements under two additional conditions. In the first condition, the observer was instructed to track the moving stimulus. In the second condition, eye movements were recorded while the observer simply fixated a stationary point with the stimulus absent.

Intentional tracking. The mean dominant orientations measured during intentional tracking are shown in the bottom row of Table 1. These orientations are very similar to the stimulus directions. Tracking eye movements to the $90^{\circ}$ and $93^{\circ}$ stimulus directions deviated, on average, just $2^{\circ}$ from those directions. In response to the $87^{\circ}$ stimulus, the dominant orientation was $79^{\circ}$, indicating an error in tracking of $8^{\circ}$ to the right. Nevertheless, the directions of the tracking eye movements were in the correct relation to the direction of the stimulus.

The distributions of tracking dominant orientations, and the magnitude of those orientations, are shown for each stimulus direction in the bottom row of Figure 3 . The 
Table 2

Mean Magnitudes of the Dominant Axial Orientations of the Eye Position Records (in Minutes of Arc)

\begin{tabular}{lccc}
\hline & \multicolumn{3}{c}{ Stimulus Direction } \\
\cline { 2 - 4 } Session & $87^{\circ}$ & $90^{\circ}$ & $93^{\circ}$ \\
\hline Beginning of & 12 & 11 & 11 \\
Training & $(3.66)$ & $(3.18)$ & $(2.64)$ \\
& {$[28]$} & {$[172]$} & {$[27]$} \\
End of & 11 & 10 & 10 \\
Training & $(7.80)$ & $(4.32)$ & $(2.82)$ \\
& {$[40]$} & {$[235]$} & {$[40]$} \\
Intentional & 96 & 100 & 122 \\
Tracking & $(30.1)$ & $(37.9)$ & $(36.2)$ \\
& {$[8]$} & {$[35]$} & {$[5]$} \\
\hline
\end{tabular}

Note-Standard deviations are shown in parentheses; the number of orientations included in each mean is shown in brackets.

dominant orientations cluster near the direction of stimulus movement. Note also the narrow distribution of the tracking eye movements on each of the three stimulus directions.

The bottom row of Table 2 shows the average magnitudes of the eye movement records taken while the subject attempted to track the stimulus. When the observer attempted to track the target, his eye moved a mean distance of $105^{\prime}$ of arc (range from 17' to $169^{\prime}$ ). This tracking distance is nearly 10 times greater than the mean magnitude of the dominant orientation during discrimination training (10.8' of arc), in which the observer was not instructed to track the stimulus.

Fixation. We then recorded eye positions while the observer was fixating a stationary target with no dots present. We compared these records to records obtained under conditions of discrimination training, in which both moving dots and a stationary fixation target were present.

In the absence of moving dots, the observer's mean dominant orientation was $159^{\circ}(S D=26.8)$. The magnitude of the dominant component during fixation was $13^{\prime}$ of arc (SD =7.45). These values are similar to the measurements obtained during training (see first two rows of Tables 1 and .2). Therefore, the observer maintained approximately the same degree of fixation whether fixating a point on an otherwise blank screen or fixating the same point superimposed on a field of moving dots.

\section{DISCUSSION}

Our results show that improvement in the discriminability of the direction in which targets move does not depend on the observer's learning to track the moving target. For one thing, the eye movements recorded during training bore little resemblance to eye movements obtained when the observer deliberately tracked the stimulus. Neither the orientation nor the magnitude of the dominant linear component extracted from the eye position records matched the direction or distance traveled by the stimulus. Also, the size and dominant orientation of eye movements were unchanged from the beginning to the end of training, although discriminability changed dramatically. In fact, both at the beginning and at the end of training, eye movements closely resembled fixation eye movements in magnitude and orientation.

Although there was a lack of tracking eye movements during training, the observer was clearly able to track the stimulus when asked to do so. In this case, the direction of tracking eye movements closely approximated the direction of the stimulus movement, and the magnitude of the tracking movements was about 10 times larger than the average magnitude of the dominant orientation of the eye position during training. This result indicates that although the observer was able to track the stimulus accurately, it was not necessary for him to track the stimulus in order to discriminate stimulus direction. These findings support the view that improvement in direction discrimination with practice is the product of a change in a visual process, rather than a change in sensorimotor response.

\section{REFERENCES}

Ball, K., \& Sekuler, R. (1982). A specific and enduring improvement in visual motion discrimination. Science, 218, 697-698.

Crane, H. D., \& Steele, C. M. (1978). Accurate three-dimensional eyetracker. Applied Optics, 17, 691-714.

Mardia, K. V. (1972). Statistics of directional data. New York: Academic Press.

Sekuler, R., Ball, K., Tynan, P., \& Machamer, J. (1982). Psychophysics of motion perception. In A. Wertheim, W. Wagenaar, \& H. W. Leibowitz (Eds.), Tutorials on motion perceprion. New York: Plenum.

SWETS, J. A. (1964). Signal detection and recognition by human observers. New York: Wiley.

\section{NOTES}

1. Earlier work (Sekuler, Ball, Tynan, \& Machamer, 1982) suggested that accuracy of direction discrimination would have reached asymptote at less than $200 \mathrm{msec}$. Therefore, the change from 512 to $640 \mathrm{msec}$ would not be expected to change performance.

2. Note that our procedure for estimating dominant orientation of the eye positions assumes that the eye's excursions can be described by a linear function. Although a record may be associated with a significant regression coefficient, this does not imply that it can be completely described by a linear model. A test of the lack of fit to a linear model shows a significant departure from linearity in the top record $[F(39,214)=2.35, p<.001]$, but not in the bottom record $[F(23,230)=0.91]$. The record in the top panel departs from linearity because it contains other, nonlinear, components. Because our main concern was to discover whether the eye moved in the same direction as the stimulus, the asumption that each record contained a significant linear component made it possible to find out if the orientation of this linear component matched the direction along which the stimulus traveled.

(Manuscript received September 3, 1985: revision accepted for publication November 19.1985.) 